Variational Sturmian approximation: A nonperturbative method of solving timeindependent Schrödinger equation

Ali Mostafazadeh

Citation: Journal of Mathematical Physics 42, 3372 (2001); doi: 10.1063/1.1385374

View online: http://dx.doi.org/10.1063/1.1385374

View Table of Contents: http://aip.scitation.org/toc/jmp/42/8

Published by the American Institute of Physics

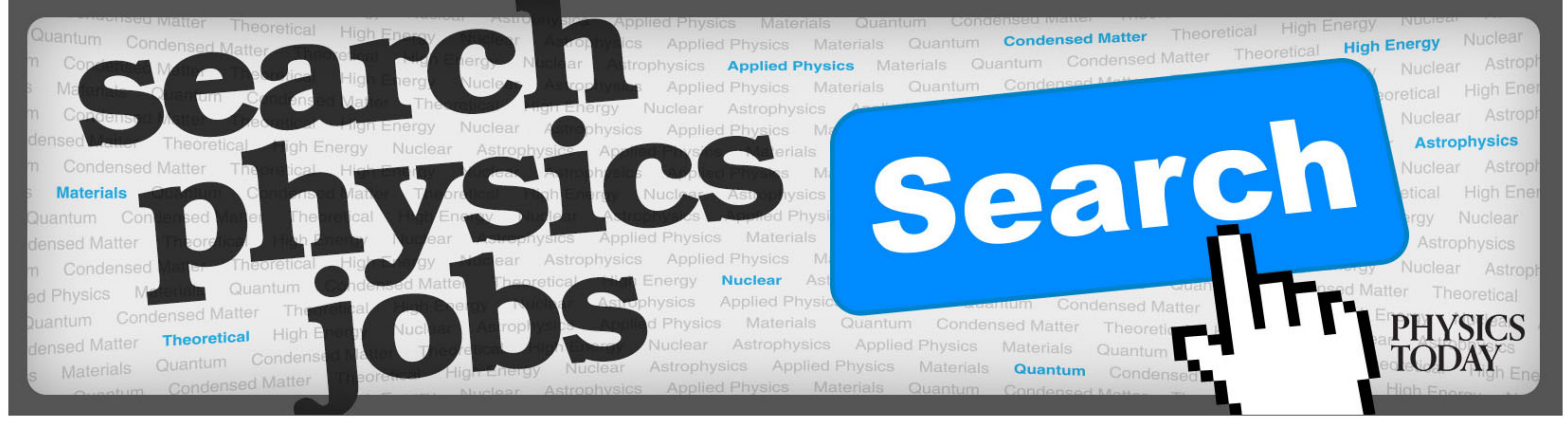




\title{
Variational Sturmian approximation: A nonperturbative method of solving time-independent Schrödinger equation
}

\author{
Ali Mostafazadeha) \\ Department of Mathematics, Koç University, \\ Rumelifeneri Yolu, 80910 Sariyer, Istanbul, Turkey
}

(Received 14 March 2001; accepted for publication 8 May 2001)

A variationally improved Sturmian approximation for solving time-independent Schrödinger equation is developed. This approximation is used to obtain the energy levels of a quartic anharmonic oscillator, a quartic potential, and a Gaussian potential. The results are compared with those of the perturbation theory, the WKB approximation, and the accurate numerical values. (C) 2001 American Institute of Physics. [DOI: 10.1063/1.1385374]

\section{INTRODUCTION}

Since the early days of quantum mechanics, the main technical tools for solving the timeindependent Schrödinger equation have been the time-independent perturbation theory, the semiclassical or WKB approximation, and the variational method. ${ }^{1,2}$ Starting form the late 1950s, physical chemists and nuclear physicists have explored the use of what is called the Sturmian basis functions in solving this equation for a variety of potentials arising in molecular and atomic physics. ${ }^{3,4}$ Recently, Antonsen ${ }^{5}$ and Szmytkowski and Zywicka-Mozeiko ${ }^{6}$ have studied the harmonic oscillator Sturmian functions. The purpose of the present paper is to outline a general variationally improved Sturmian approximation scheme that provides a nonperturbative method of solving time-independent Schrödinger equation.

The organization of the paper is as follows. In Sec. II, we give the definition of the Sturmian basis vectors, derive their general properties, and discuss the conventional Sturmian approximation. In Sec. III we present an improved Sturmian approximation which makes use of the variational method. In Sec. IV we study the harmonic oscillator Sturmians and use them for the solution of time-independent Schrödinger equation in one dimension. In Sec. V we apply our general results to some concrete problems, and compare our results with those obtained using perturbation theory, the WKB approximation, and the highly accurate numerical investigations. In particular, we obtain the energy levels of a quartic anharmonic oscillator, a quartic potential, and a Gaussian potential. Finally, in Sec. VI we summarize our results and present our conclusions.

\section{CONVENTIONAL STURMIAN APPROXIMATION}

Consider the time-independent Schrödinger equation:

$$
H|E, a\rangle=E|E, a\rangle
$$

where $H$ is a self-adjoint Hamiltonian operator and $a$ is a degeneracy label.

The method of Sturmian approximation is based on an expansion of the eigenvectors $|E, a\rangle$ in terms of solutions $\left|\phi_{\nu}, \alpha\right\rangle$ of the equation

$$
\left(H_{0}+\beta_{\nu} V_{0}\right)\left|\phi_{\nu}, \alpha\right\rangle=\mathcal{E}\left|\phi_{\nu}, \alpha\right\rangle,
$$

${ }^{a)}$ Electronic mail: amostafazadeh@ku.edu.tr 
where $H_{0}$ and $V_{0}$ are self-adjoint operators, $\beta_{\nu}$ and $\mathcal{E}$ are real scalar parameters, and $\alpha$ is a degeneracy label. Note that in order to obtain $\left|\phi_{\nu}, \alpha\right\rangle$, one must fix $\mathcal{E}$ and solve Eq. (2) for $\left|\phi_{\nu}, \alpha\right\rangle$. Clearly, every solution $\left|\phi_{\nu}, \alpha\right\rangle$ would correspond to a choice for the value of the coupling constant $\beta_{\nu}$.

Suppose that $H_{0}=\vec{p}^{2} /(2 m)$ is the Hamiltonian for a free particle moving in the configuration space $\mathbb{R}^{d}, V_{0}=V_{0}(\vec{x})$ is a real interaction potential, and $H$ is a standard Hamiltonian of the form

$$
H=\frac{\vec{p}^{2}}{2 m}+V(\vec{x})
$$

where $\vec{p}$ and $\vec{x}$ are momentum and position operators, respectively. If $V_{0}(\vec{x})$ tends to infinity as $|\vec{x}| \rightarrow \infty$, all the eigenstates of $V_{0}$ are bound states. ${ }^{7}$ In this case, only for a discrete set of positive values of $\beta_{\nu}$, can we find square-integrable solutions $\left|\phi_{\nu}, \alpha\right\rangle$ of Eq. (2). In this case, the label $\nu$ will take values in a discrete set which we shall choose to be $\{0,1,2, \ldots\}$.

The only difference between Eq. (2) and the eigenvalue equation for the potential $\beta_{\nu} V_{0}(\vec{x})$ is that in the former $\mathcal{E}$ is a fixed parameter which can be arbitrarily chosen. Therefore, a solution of Eq. (2) corresponds to a pair $\left(\beta_{\nu},\left|\phi_{\nu}, \alpha\right\rangle\right)$.

The vectors $\left|\phi_{\nu}, \alpha\right\rangle$ are called the Sturmian basis vectors or simply the Sturmians. ${ }^{3}$ They satisfy certain orthonormality conditions which we shall derive below. We should, however, note that the square-integrable Sturmians do not generally constitute a complete set of basis vectors of the Hilbert space. ${ }^{6}$ There are certain potentials $V_{0}$, such as the Coulomb potential, that lead to a complete set of square-integrable Sturmians. ${ }^{8}$

Let us first note that the defining equation (2) does not determine $\left|\phi_{\nu}, \alpha\right\rangle$ uniquely. This is reflected in the presence of the degeneracy label $\alpha$. What is uniquely determined by Eq. (2) is the degeneracy subspace $\mathcal{H}_{\nu}$ spanned by $\left\{\left|\phi_{\nu}, 1\right\rangle,\left|\phi_{\nu}, 2\right\rangle, \ldots,\left|\phi_{\nu}, l_{\nu}\right\rangle\right\}$, where $l_{\nu}$ is the degree of degeneracy, i.e., the number of linearly independent solutions of Eq. (2) associated with a given (admissible) value of $\beta_{\nu}$. Clearly, we can construct an orthonormal basis of $\mathcal{H}_{\nu}$ and choose the Sturmian vectors $\left|\phi_{\nu}, \alpha\right\rangle$ to be the basis vectors. In other words, without loss of generality, we can choose to work with the Sturmians $\left|\phi_{\nu}, \alpha\right\rangle$ satisfying

$$
\left\langle\phi_{\nu}, \alpha \mid \phi_{\nu}, \gamma\right\rangle=\delta_{\alpha \gamma},
$$

where $\delta_{\alpha \gamma}$ denotes the Kronecker delta function. Clearly, any unitary transformation of $\mathcal{H}_{\nu}$ would lead to a new set of Sturmians satisfying (4). Therefore, the condition (4) reduces the freedom in the choice of the Sturmians $\left|\phi_{\nu}, \alpha\right\rangle$, but does not eliminate it.

Next, we evaluate the Hermitian adjoint of both sides of Eq. (2), change $(\nu, \alpha)$ to $(\mu, \gamma)$, and take the inner product of both sides of the resulting equation with $\left|\phi_{\nu}, \alpha\right\rangle$. This yields

$$
\beta_{\mu}\left\langle\phi_{\mu}, \gamma\left|V_{0}\right| \phi_{\nu}, \alpha\right\rangle=\left\langle\phi_{\mu}, \gamma\left|\left(\mathcal{E}-H_{0}\right)\right| \phi_{\nu}, \alpha\right\rangle \text {. }
$$

We can compute the right-hand side of this equation using Eq. (2). Substituting the result in (5), we find

$$
\left(\beta_{\mu}-\beta_{\nu}\right)\left\langle\phi_{\mu}, \gamma\left|V_{0}\right| \phi_{\nu}, \alpha\right\rangle=0
$$

If we define

$$
N_{\nu}^{\gamma \alpha}:=\left\langle\phi_{\nu}, \gamma\left|V_{0}\right| \phi_{\nu}, \alpha\right\rangle,
$$

then we can write Eq. (6) in the form

$$
\left\langle\phi_{\mu}, \gamma\left|V_{0}\right| \phi_{\nu}, \alpha\right\rangle=N_{\nu}^{\gamma \alpha} \delta_{\mu \nu} .
$$


Equation (8) is the desired orthogonality property of the Sturmians. We can further simplify Eq. (8), by noting that the $l_{\nu} \times l_{\nu}$ matrix $N_{\nu}$ formed by $N_{\nu}^{\gamma \alpha}$ is a Hermitian matrix. This means that we can choose $\left|\phi_{\nu}, \alpha\right\rangle$ in such a way that $N_{\nu}$ is a diagonal matrix. Making this choice, we have

$$
\begin{gathered}
N_{\nu}^{\alpha \gamma}=N_{\nu}^{\alpha} \delta_{\alpha \gamma}, \\
\left\langle\phi_{\mu}, \gamma\left|V_{0}(\vec{x})\right| \phi_{\nu}, \alpha\right\rangle=N_{\nu}^{\alpha} \delta_{\mu \nu} \delta_{\alpha \gamma},
\end{gathered}
$$

where $N_{\alpha}^{\nu}$, with $\alpha \in\left\{1,2, \ldots, l_{\nu}\right\}$, are eigenvalues of the matrix $N_{\nu}$. Since $N_{\nu}$ is Hermitian, $N_{\nu}^{\alpha}$ are real.

In summary, we can choose a set of Sturmian vectors $\left|\phi_{\nu}, \alpha\right\rangle$ which are eigenvectors of the matrices $N_{\nu}$. Therefore, for each value of $\nu,\left\{\left|\phi_{\nu}, 1\right\rangle, \ldots,\left|\phi_{\nu}, l_{\nu}\right\rangle\right\}$ forms an orthonormal eigenbasis of $N_{\nu}$ in the degeneracy subspace $\mathcal{H}_{\nu}$. However, $\left|\phi_{\nu}, \alpha\right\rangle$ with different values of $\nu$ are not orthogonal. Instead, they satisfy a modified orthogonality condition, namely (10).

Now, let us expand a solution $|E, a\rangle$ of the Schrödinger equation (1), in a Sturmian basis corresponding to a solvable potential $V_{0}$, i.e., seek solutions of the form

$$
|E, a\rangle=\sum_{\nu=0}^{\infty} \sum_{\alpha=1}^{l_{\nu}} C_{\nu}^{\alpha}\left|\phi_{\nu}, \alpha\right\rangle
$$

where $C_{\nu}^{\alpha}$ are complex coefficients and $\nu$ is supposed to take discrete values $0,1,2, \ldots$. Note that if the Sturmians $\left|\phi_{\nu}, \alpha\right\rangle$ do not form a complete basis, then Eq. (11) yields the eigenvectors that belong to the span of $\left|\phi_{\nu}, \alpha\right\rangle$.

The Sturmian approximation of order $N$ is the approximation in which one neglects all the coefficients $C_{\nu}^{\alpha}$ in Eq. (11) but those with $\nu$ belonging to a subset $\mathcal{S}_{N+1}$ of non-negative integers of order $N+1$. Alternatively, in considering the Sturmian approximation of order $N$, one confines the range of the indices (of type) $\nu$ to a fixed finite set $\mathcal{S}_{N+1}$. In this way, the infinite sum $\Sigma_{\nu=0}^{\infty} \cdots$ in Eq. (11) is replaced by the finite sum $\Sigma_{\nu \in \mathcal{S}_{N+1}} \cdots$. We shall abbreviate the latter by $\Sigma_{\nu}$. The set $\mathcal{S}_{N+1}$ may, in principle, be chosen arbitrarily. We will comment on this choice in Sec. III.

Substituting (11) in the Schrödinger equation (1) and making use of Eqs. (2) and (3), we find

$$
\sum_{\nu} \sum_{\alpha=1}^{l_{\nu}} C_{\nu}^{\alpha}\left(E-\mathcal{E}-V+\beta_{\nu} V_{0}\right)\left|\phi_{\nu}, \alpha\right\rangle=0 .
$$

Now, evaluating the inner product of both sides of this equation with $\left|\phi_{\mu}, \gamma\right\rangle$ and using the orthogonality relation (10), we obtain

$$
\sum_{\nu} \sum_{\alpha=1}^{l_{\nu}}\left[(E-\mathcal{E}) T_{\mu \nu}^{\gamma \alpha}-\left(W_{\mu \nu}^{\gamma \alpha}-\beta_{\nu} N_{\nu}^{\alpha} \delta_{\mu \nu} \delta_{\gamma \alpha}\right)\right] C_{\nu}^{\alpha}=0 .
$$

Here we have introduced

$$
\begin{aligned}
T_{\mu \nu}^{\gamma \alpha} & :=\left\langle\phi_{\mu}, \gamma \mid \phi_{\nu}, \alpha\right\rangle, \\
W_{\mu \nu}^{\gamma \alpha}: & =\left\langle\phi_{\mu}, \gamma|V| \phi_{\nu}, \alpha\right\rangle .
\end{aligned}
$$

We can express Eq. (13) in a more compact form, if we use a single label for the pair $(\nu, \alpha)$. Introducing $\mathcal{N}:=(\nu, \alpha)$ and $\mathcal{M}:=(\mu, \gamma)$, we write Eq. (13) in the form

$$
\sum_{\mathcal{N}}\left[(E-\mathcal{E}) T_{\mathcal{M N}}-S_{\mathcal{M N}}\right] C_{\mathcal{N}}=0
$$

where 


$$
\begin{gathered}
T_{\mathcal{M N}}=T_{\mu \nu}^{\gamma \alpha}, \quad S_{\mathcal{M N}}=W_{\mathcal{M N}}-\beta_{\nu} N_{\mathcal{N}} \delta_{\mathcal{M N}} \\
W_{\mathcal{M N}}=W_{\mu \nu}^{\gamma \alpha}, \quad N_{\mathcal{N}}=N_{\nu}^{\alpha} .
\end{gathered}
$$

Equations (16) form a linear system of homogeneous first order algebraic equations for $C_{\mathcal{N}}$. This system has a nontrivial solution provided that the determinant of the matrix of coefficients vanishes, i.e.,

$$
\operatorname{det}[(E-\mathcal{E}) T-S]=0 .
$$

Here $T$ and $S$ are matrices with entries $T_{\mathcal{M N}}$ and $S_{\mathcal{M N}}$, respectively.

Solving Eq. (19), we can express $E$ in terms of $\mathcal{E}, \beta_{\nu}$, and the Sturmians $\left|\phi_{\nu}, \alpha\right\rangle$. Now, we recall that for a fixed choice of $V_{0}$, the coupling constants $\beta_{\nu}$ and the corresponding Sturmians $\left|\phi_{\nu}, \alpha\right\rangle$ depend on the parameter $\mathcal{E}$. Therefore, Eq. (19) yields $E$ as a function of $\mathcal{E}$. Furthermore, substituting the value of $E=E(\mathcal{E})$ obtained by solving (19) in (16) and solving for the coefficients $C_{\mathcal{N}}$, we obtain an expression for the eigenvector $|E, a\rangle$ that involves $\mathcal{E}$. As we discuss in the following section, the fact that the Sturmian approximation yields the eigenvalues and the eigenvectors of the Hamiltonian as functions of a free parameter seems to have been overlooked. This is mainly because there is a choice for the parameter $\mathcal{E}$ that simplifies the calculations.

It should also be emphasized that Eq. (19) is an algebraic equation of order $N+1$. Therefore, in general, it has $N+1$ solutions. This can be understood by noting that in the Sturmian approximation one actually approximates the Hilbert space by a finite-dimensional vector space. Consequently, the Hamiltonian is replaced with a matrix with a finite number of eigenvalues.

\section{VARIATIONAL STURMIAN APPROXIMATION}

In general, the accuracy of the Sturmian approximation depends on the following factors.

(1) Choice of $V_{0}$ : In practice, $V_{0}$ must be one of the exactly solvable potentials. Therefore, the available choices for $V_{0}$ are few in number. For the potentials $V$ with bound states, we can choose $V_{0}$ to be a harmonic oscillator potential. For example, for the quartic anharmonic oscillator

$$
V(x)=\frac{k}{2} x^{2}+\epsilon x^{4},
$$

we shall take

$$
V_{0}(x)=\frac{k}{2} x^{2} .
$$

Similarly, for the Gaussian potential

$$
V(x)=-\lambda e^{-\epsilon x^{2} / 2},
$$

we shall take

$$
V_{0}(x)=\frac{1}{2} \lambda \epsilon x^{2}-\lambda .
$$

This will enable us to compare the results of the Sturmian approximation with those of the perturbation theory, for in the limit $\epsilon \rightarrow 0, V(r) \rightarrow V_{0}(r)$. Note that multiplying $V_{0}$ by a positive real number does not change the results of the Sturmian approximation. This is simply because we can always absorb such a number in the definition of $\beta_{\nu}$.

(2) Choice of the Sturmians included in the sum (16): This is also directly related to the choice of the potential $V_{0}$. If $V_{0}$ is obtained from $V$ by a limiting process as in the case of the potentials (20) 
and (22), then a natural choice for the computation of the $n$th energy eigenvalue $E_{n}$ and the corresponding eigenvectors $\left|E_{n}, \alpha\right\rangle$ is to include the $\left|\phi_{\nu}, \alpha\right\rangle$ with $\nu$ equal or close to $n$. In particular, in the Sturmian approximation of order zero, we have

$$
\left|E_{n}, a\right\rangle=\sum_{\alpha=1}^{l_{n}} C_{n}^{\alpha}\left|\phi_{n}, \alpha\right\rangle .
$$

(3) Choice of the parameter $\mathcal{E}$ : The conventional choice ${ }^{4,5}$ for the parameter $\mathcal{E}$ is $\mathcal{E}=E$. This simplifies Eq. (19) considerably. The basic idea pursued in this paper is the fact that this simplification does not necessarily justify the conventional choice for $\mathcal{E}$.

It is well known ${ }^{2}$ that the eigenvalue equation (1) is equivalent to the variational equation

$$
\frac{\delta}{\delta\langle\psi|}\left(\frac{\langle\psi|H| \psi\rangle}{\langle\psi \mid \psi\rangle}\right)=0 .
$$

In other words, the eigenvalues $E$ are the minima of the expectation value

$$
\langle H\rangle:=\frac{\langle\psi|H| \psi\rangle}{\langle\psi \mid \psi\rangle},
$$

and the eigenvectors are the vectors $|E\rangle=|E, a\rangle$ that minimize $\langle H\rangle$. This observation suggests that the most efficient choice for the parameter $\mathcal{E}$ appearing in the Sturmian approximation is the one that minimizes $E=E(\mathcal{E})$. Therefore, the most reliable Sturmian approximation is obtained by choosing $\mathcal{E}$ to be a solution of

$$
\frac{d E}{d \mathcal{E}}=0 .
$$

If this equation does not have a solution, then one must either make another choice for the set $\mathcal{S}_{N+1}$ or proceed with a higher order Sturmian approximation.

\section{VARIATIONAL STURMIAN APPROXIMATION USING HARMONIC OSCILLATOR STURMIANS}

Consider a quantum system with the configuration space R, a standard Hamiltonian (3), and a real-valued potential $V=V(x)$. Suppose that the system has an infinite number of bound states with nondegenerate energy eigenvalues $E_{n}$. Here $n \in\{0,1,2, \ldots\}$ and $E_{0}$ stands for the ground state.

Now, consider the Sturmian basis vectors associated with a harmonic oscillator ${ }^{5,6}$

$$
V_{0}=V_{0}(x)=\frac{k}{2} x^{2} .
$$

In order to solve Eq. (2) for this choice of $V_{0}$, we introduce

$$
\begin{gathered}
\omega_{\nu}:=\left(\frac{\beta_{\nu} k}{m}\right)^{1 / 2}, \\
\alpha_{\nu}:=\frac{m \omega_{\nu}}{\hbar}, \\
a_{\nu}:=\left(\frac{\alpha_{\nu}}{2}\right)^{1 / 2}\left(x+\frac{i p}{\hbar \alpha_{\nu}}\right) .
\end{gathered}
$$




$$
|l\rangle_{\nu}:=\frac{1}{\sqrt{l} !} a_{\nu}^{\dagger l}|0\rangle_{\nu}
$$

where $|0\rangle_{\nu}$ is the normalized real ground state vector for a harmonic oscillator with mass $m$ and frequency $\omega_{\nu}$. That is

$$
\langle x \mid 0\rangle_{\nu}:=\left(\frac{\alpha_{\nu}}{\pi}\right)^{1 / 4} e^{-\alpha_{\nu} x^{2} / 2} .
$$

In view of the similarity of Eq. (2) with the eigenvalue equation for the potential $\beta_{\nu} V_{0}$, we can easily deduce that

$$
\begin{gathered}
\mathcal{E}=\hbar \omega_{\nu}\left(\nu+\frac{1}{2}\right), \\
\left|\phi_{\nu}\right\rangle=|\nu\rangle_{\nu}
\end{gathered}
$$

where $\nu \in\{0,1,2, \ldots\}$.

We can invert Eqs. (33) and (28) to express $\omega_{\nu}$ and $\beta_{\nu}$ in terms of $\mathcal{E}$. This yields

$$
\begin{gathered}
\omega_{\nu}=\frac{2 \mathcal{E}}{\hbar(2 \nu+1)}, \\
\beta_{\nu}=\frac{4 m \mathcal{E}^{2}}{\hbar^{2} k(2 \nu+1)^{2}} .
\end{gathered}
$$

Substituting Eq. (35) in (29), we have

$$
\alpha_{\nu}=\frac{2 m \mathcal{E}}{\hbar^{2}(2 \nu+1)}=\frac{\alpha_{0}}{2 \nu+1} .
$$

Next, we compute the term $\beta_{\nu} N_{\nu}=\beta_{\nu}\left\langle\phi_{\nu}\left|V_{0}\right| \phi_{\nu}\right\rangle$. We can use the properties of the annihilation operator $a_{\nu}$, namely

$$
a_{\nu}|l\rangle_{\nu}=\sqrt{l}|l-1\rangle_{\nu}, \quad a_{\nu}^{\dagger}|l\rangle_{\nu}=\sqrt{l+1}|l+1\rangle_{\nu}, \quad x=\left(2 \alpha_{\nu}\right)^{-1 / 2}\left(a_{\nu}+a_{\nu}^{\dagger}\right),
$$

and the orthonormality of $|l\rangle_{\nu}$ to compute

$$
{ }_{\nu}\left\langle l\left|x^{2}\right| \nu\right\rangle_{\nu}=\left(2 \alpha_{\nu}\right)^{-1}\left[(2 \nu+1) \delta_{l, \nu}+\sqrt{(\nu+1)(\nu+2)} \delta_{l, \nu+2}+\sqrt{\nu(\nu-1)} \delta_{l, \nu-2}\right] .
$$

In view of Eqs. (27) and (39), we obtain, after some remarkable simplifications,

$$
\beta_{\nu} N_{\nu}=\frac{\mathcal{E}}{2} .
$$

\section{A. Variational Sturmian approximation of order zero}

For the variational Sturmian approximation of order zero, $\nu=n$, and Eq. (19) takes the form

$$
\left(E_{n}-\mathcal{E}\right) T-S=0
$$

where

$$
T=\left\langle\phi_{n} \mid \phi_{n}\right\rangle=1, \quad S=W-\beta_{n} N_{n}, \quad W=\left\langle\phi_{n}|V| \phi_{n}\right\rangle .
$$

According to Eqs. (41), (42), and (40), the energy eigenvalues $E_{n}$ are given by 


$$
E_{n}=W+\frac{\mathcal{E}}{2}
$$

Next, we fix the parameter $\mathcal{E}$ using Eq. (26). This requires the computation of $d W / d \mathcal{E}$. We first evaluate the variation of $W$,

$$
\delta W=\left(\delta\left\langle\phi_{n}\right|\right) V\left|\phi_{n}\right\rangle+\left\langle\phi_{n}\right| V\left(\delta\left|\phi_{n}\right\rangle\right)=2\left\langle\phi_{n}\right| V\left(\delta\left|\phi_{n}\right\rangle\right)=2 \sum_{l=0}^{\infty}{ }_{n}\langle n|V| l\rangle_{n}\langle l|\left(\delta|n\rangle_{n}\right) .
$$

Here we have made use of Eq. (34), the fact that the Sturmians and the potential $V$ are real and $|l\rangle_{n}$ form a complete set of basis vectors.

We can compute ${ }_{n}\langle l|\left(\delta|n\rangle_{n}\right)$ using the eigenvalue equation

$$
\left(H_{0}+\beta_{n} V_{0}\right)|j\rangle_{n}=\mathcal{E}_{j}|j\rangle_{n},
$$

where $\mathcal{E}_{j}=\hbar \omega_{n}(j+1 / 2)$. Taking the variation of both sides of this equation and computing the inner product with $|l\rangle_{n}$, we find

$$
{ }_{n}\langle l|\delta| j\rangle_{n}=\frac{{ }_{n}\left\langle l\left|V_{0}\right| j\right\rangle_{n}\left(\delta \beta_{n}\right)}{\mathcal{E}_{j}-\mathcal{E}_{l}} \text { for } l \neq j .
$$

Furthermore, using the fact that the eigenfunctions $\langle x \mid l\rangle_{n}$ are real, we can easily show that

$$
{ }_{n}\langle l|\left(\delta|l\rangle_{n}\right)=0 .
$$

Equations (44), (46) and (47) reduce the computation of $\delta W$ to that of

$$
{ }_{n}\left\langle l\left|V_{0}\right| n\right\rangle_{n}=\frac{k}{2}{ }_{n}\left\langle l\left|x^{2}\right| n\right\rangle_{n} .
$$

We have already computed ${ }_{n}\left\langle l\left|x^{2}\right| n\right\rangle_{n}$ in Eq. (39). Substituting this equation in Eq. (48) and using Eqs. (46) and (44), we find, after some remarkable cancellations,

$$
\delta W=\left(\frac{\delta \mathcal{E}}{2 \mathcal{E}}\right)\left[\sqrt{n(n-1)}_{n}\langle n|V| n-2\rangle_{n}-\sqrt{(n+1)(n+2)}_{n}\langle n|V| n+2\rangle_{n}\right] .
$$

Now, in view of Eqs. (43) and (49),

$$
\frac{d E_{n}}{d \mathcal{E}}=\left(\frac{1}{2 \mathcal{E}}\right)\left[\sqrt{n(n-1)}{ }_{n}\langle n|V| n-2\rangle_{n}-\sqrt{(n+1)(n+2)}\left\langle\langle|V| n+2\rangle_{n}\right]+\frac{1}{2} .\right.
$$

Substituting this equation in Eq. (26) yields

$$
\mathcal{E}=\sqrt{(n+1)(n+2)}{ }_{n}\langle n|V| n+2\rangle_{n}-\sqrt{n(n-1)}_{n}\langle n|V| n-2\rangle_{n} .
$$

Note that the right-hand side of this equation also involves $\mathcal{E}$. This is because $|l\rangle_{n}$ depend on $\mathcal{E}$.

Using Eqs. (50) and (43) we can express the energy eigenvalue $E_{n}$ in terms of $V$. This yields

$$
E_{n}={ }_{n}\langle n|V| n\rangle_{n}+\frac{1}{2}\left[\sqrt{(n+1)(n+2)}_{n}\langle n|V| n+2\rangle_{n}-\sqrt{n(n-1)}_{n}\langle n|V| n-2\rangle_{n}\right] .
$$

For the ground state $n=0$ and Eq. (51) reduces to

$$
E_{0}={ }_{0}\langle 0|V| 0\rangle_{0}+\frac{1}{\sqrt{2}}{ }_{0}\langle 0|V| 2\rangle_{0}
$$


Note that the vectors $|l\rangle_{n}$ appearing in Eqs. (51) and (52) are those of Eq. (31) with $\mathcal{E}$ being a solution of Eq. (50).

Equations (51) and (52) are of limited importance. In practice, one obtains the energy eigenvalue $E_{n}$ by substituting the solution of Eq. (50) in Eq. (43).

The variational Sturmian approximation of order zero, as outlined above, is a valid approximation scheme, if Eq. $(50)$ has a unique positive solution $\mathcal{E}$. If such a solution does not exist, one may attempt to construct higher order variational Sturmian approximations. As we shall see in Sec. $\mathrm{V}$, for all the potentials that we have considered, Eq. (50) has a unique positive solution. This is very remarkable, for this equation turns out to be an algebraic equation of order three for the quartic anharmonic oscillator and the quartic potential, and of order four for the Gaussian potential.

\section{B. Variational Sturmian approximation of order one}

In the variational sturmian approximation of order one, the number of Sturmians contributing to the eigenvector $|E\rangle$ is two. We shall denote them by $\left|\phi_{n}\right\rangle$ and $\left|\phi_{m}\right\rangle$.

The matrices $T$ and $W$ are Hermitian $2 \times 2$ matrices. They can be written in the form

$$
T=\left(\begin{array}{cc}
1 & t^{*} \\
t & 1
\end{array}\right), \quad W=\left(\begin{array}{cc}
v_{n} & w^{*} \\
w & v_{m}
\end{array}\right),
$$

where we have used the fact that the Sturmians are normalized and introduced

$$
t:=\left\langle\phi_{m} \mid \phi_{n}\right\rangle, \quad v_{\nu}:=\left\langle\phi_{\nu}|V| \phi_{\nu}\right\rangle, \quad w:=\left\langle\phi_{m}|V| \phi_{n}\right\rangle .
$$

Next, we construct the matrix $S$. In view of Eqs. (17) and (53),

$$
S=\left(\begin{array}{cc}
v_{n}-\beta_{n} N_{n} & w^{*} \\
w & v_{m}-\beta_{m} N_{m}
\end{array}\right) .
$$

Note that because the Sturmians for the harmonic oscillator are real-valued, $t$ and $w$ are real-valued functions of the parameter $\mathcal{E}$. In particular, the matrices $T, W$, and $S$ are real and symmetric.

Substituting Eqs. (53) and (55) in the Eq. (19), making use of Eq. (40), and simplifying the resulting expression, we find

$$
A\left(E-\frac{\mathcal{E}}{2}\right)^{2}+B\left(E-\frac{\mathcal{E}}{2}\right)+C=0
$$

where

$$
A:=1-t^{2}, \quad B:=t(t \mathcal{E}+2 w)-\left(v_{n}+v_{m}\right), \quad C:=v_{n} v_{m}-\left(\frac{t \mathcal{E}}{2}+w\right)^{2} .
$$

Note that the coefficients $A, B$, and $C$ are functions of $\mathcal{E}$. Equation (56) can be easily solved to express $E$ in terms of $\mathcal{E}$. The result is

$$
E=E_{ \pm}:=\frac{\mathcal{E}}{2}+\frac{-B \pm \sqrt{B^{2}-4 A C}}{2 A} .
$$

The next step is to determine $\mathcal{E}$ using the variational principle, i.e., setting $d E / d \mathcal{E}=0$. The resulting formulas are complicated and we shall not include them here.

We conclude this section with the following remarks. 
(1) As seen from Eq. (58), the first order Sturmian approximation leads to a pair of energy eigenvalues. For the potentials which are related to $V_{0}$ via a limiting process, one expects these two eigenvalues to be those labeled by $m$ and $n$. That is, for $n<m$,

$$
E_{n}=E_{-}, \quad E_{m}=E_{+} .
$$

(2) In the variational Sturmian approximation of order one, there are two variational equations $d E_{ \pm} / d \mathcal{E}=0$. It is not clear whether these equations lead to a unique minimum for $E_{ \pm}(\mathcal{E})$ with a positive value for $\mathcal{E}$. As we shall show in the following sections, for all the specific examples that we have considered each of these equations lead to a unique minimum with a positive value for $\mathcal{E}$. Lack of such a solution may be interpreted as the failure of the variational Sturmian approximation of order one.

\section{Variational Sturmian approximation of order two}

In the variational Sturmian approximation of order two, one uses three Sturmians to expand the energy eigenvectors $|E\rangle$. We shall denote these by $\left|\phi_{n_{l}}\right\rangle$ where $n_{l} \in \mathcal{S}_{3}:=\left\{n_{1}, n_{2}, n_{3}\right\}$.

The matrices $T, W$, and $S$ are given by

$$
\begin{gathered}
T=\left(\begin{array}{ccc}
1 & t_{1}^{*} & t_{2}^{*} \\
t_{1} & 1 & t_{3}^{*} \\
t_{2} & t_{3} & 1
\end{array}\right), \quad W=\left(\begin{array}{ccc}
v_{3} & w_{1}^{*} & w_{2}^{*} \\
w_{1} & v_{2} & w_{3}^{*} \\
w_{2} & w_{3} & v_{1}
\end{array}\right), \\
S=\left(\begin{array}{ccc}
v_{3}-\beta_{n_{3}} N_{n_{3}} & w_{1}^{*} & w_{2}^{*} \\
w_{1} & v_{2}-\beta_{n_{2}} N_{n_{2}} & w_{3}^{*} \\
w_{2} & w_{3} & v_{1}-\beta_{n_{1}} N_{n_{1}}
\end{array}\right),
\end{gathered}
$$

where we have used the fact that $\left|\phi_{n_{l}}\right\rangle$ are normalized and introduced

$$
\begin{gathered}
t_{1}:=\left\langle\phi_{n_{2}} \mid \phi_{n_{3}}\right\rangle, \quad t_{2}:=\left\langle\phi_{n_{1}} \mid \phi_{n_{3}}\right\rangle, \quad t_{3}:=\left\langle\phi_{n_{1}} \mid \phi_{n_{2}}\right\rangle, \quad v_{l}:=\left\langle\phi_{n_{l}}|V| \phi_{n_{l}}\right\rangle, \\
w_{1}:=\left\langle\phi_{n_{2}}|V| \phi_{n_{3}}\right\rangle, \quad w_{2}:=\left\langle\phi_{n_{1}}|V| \phi_{n_{3}}\right\rangle, \quad w_{3}:=\left\langle\phi_{n_{1}}|V| \phi_{n_{2}}\right\rangle .
\end{gathered}
$$

Because the harmonic oscillator Sturmian functions are real-valued, $t_{l}, v_{l}$, and $w_{l}$ are real, and $T$, $W$, and $S$ are real symmetric matrices.

In view of Eq. (40), we can write the secular equation (19) in the form

$$
A\left(E-\frac{\mathcal{E}}{2}\right)^{3}+B\left(E-\frac{\mathcal{E}}{2}\right)^{2}+C\left(E-\frac{\mathcal{E}}{2}\right)+D=0
$$

where

$$
\begin{gathered}
A:=1-\sum_{l=1}^{3} t_{l}^{2}+2 t_{1} t_{2} t_{3}, \\
B:=\sum_{l=1}^{3}\left[\left(t_{l}^{2}-1\right) v_{l}+2 t_{l} \xi_{l}\right]-2\left(t_{1} t_{2} \xi_{3}+t_{3} t_{1} \xi_{2}+t_{2} t_{3} \xi_{1}\right), \\
C:=v_{1} v_{2}+v_{2} v_{3}+v_{3} v_{1}+2\left(t_{1} \xi_{2} \xi_{3}+t_{3} \xi_{1} \xi_{2}+t_{2} \xi_{3} \xi_{1}\right)-\sum_{l=1}^{3}\left(\xi_{l}^{2}+2 t_{l} v_{l} \xi_{l}\right),
\end{gathered}
$$




$$
\begin{gathered}
D:=\sum_{l=1}^{3} v_{l} \xi_{l}^{2}-2 \xi_{1} \xi_{2} \xi_{3}-v_{1} v_{2} V_{3}, \\
\xi_{l}:=\frac{1}{2} t_{l} \mathcal{E}+w_{l} .
\end{gathered}
$$

Equation (64) has, in general, three solutions. The desired eigenvalues are the minima of these solutions corresponding to positive values of $\mathcal{E}$. Again for the cases where $V$ is related to $V_{0}$ by a limiting process the minima of the solutions of Eq. (64) correspond to $E_{n_{1}}, E_{n_{2}}$, and $E_{n_{3}}$.

\section{APPLICATIONS}

In this section, we apply our general results to compute the energy eigenvalues of a quartic anharmonic oscillator, a quartic potential, and a Gaussian potential.

\section{A. The quartic anharmonic oscillator}

Consider the potential

$$
V(x)=\frac{k}{2} x^{2}+\epsilon x^{4}
$$

In order to obtain the energy levels of this potential using variational Sturmian approximation of order zero, we need to calculate ${ }_{n}\langle n|V| l\rangle_{n}$. We first use Eqs. (38) to compute

$$
\begin{aligned}
{ }_{n}\left\langle n\left|x^{4}\right| l\right\rangle_{n}= & \left(2 \alpha_{n}\right)^{-2}\left[3\left(2 n^{2}+2 n+1\right) \delta_{l, n}+4(n+1) \sqrt{(n+1)(n+2)} \delta_{l, n+2}\right. \\
& +2(2 n-1) \sqrt{n(n-1)} \delta_{l, n-2}+\sqrt{(n+1)(n+2)(n+3)(n+4)} \delta_{l, n+4} \\
& \left.+\sqrt{(n-3)(n-2)(n-1) n} \delta_{l, n-4}\right] .
\end{aligned}
$$

In view of this equation and Eqs. (39) and (70),

$$
\begin{gathered}
W={ }_{n}\langle n|V| n\rangle_{n}=\frac{(2 n+1) k}{4 \alpha_{n}}+\frac{3\left(2 n^{2}+2 n+1\right) \epsilon}{4 \alpha_{n}^{2}}, \\
{ }_{n}\langle n|V| n+2\rangle_{n}=\sqrt{(n+1)(n+2)}\left(\frac{k}{4 \alpha_{n}}+\frac{(n+1) \epsilon}{\alpha_{n}^{2}}\right), \\
{ }_{n}\langle n|V| n-2\rangle_{n}=\sqrt{n(n-1)}\left(\frac{k}{4 \alpha_{n}}+\frac{(2 n-1) \epsilon}{2 \alpha_{n}^{2}}\right),
\end{gathered}
$$

Next, we substitute Eqs. (73) and (74) in Eq. (50). Using Eq. (37), we then obtain

$$
\mathcal{E}^{3}-p_{n} \mathcal{E}-q_{n}=0
$$

where

$$
p_{n}:=\left(\frac{\hbar^{2} k}{m}\right)\left(n+\frac{1}{2}\right)^{2}, \quad q_{n}:=\left(\frac{\hbar^{4} \epsilon}{2 m^{2}}\right)\left(n+\frac{1}{2}\right)^{2}\left(11 n^{2}+9 n+4\right) .
$$

It is not difficult to show that Eq. (75) has a single positive solution [this is true for any positive $p_{n}$ and $q_{n}$ ] given by ${ }^{9}$

$$
\mathcal{E}=\left(\frac{q_{n}}{2}\right)^{1 / 3}\left(1+\sqrt{1-r_{n}}\right)^{1 / 3}+\left(\frac{p_{n}}{3}\right)\left(\frac{2}{q_{n}}\right)^{1 / 3}\left(1+\sqrt{1-r_{n}}\right)^{-1 / 3},
$$


TABLE I. First 10 energy levels of the Hamiltonian $H=p^{2}+x^{2}+\left(x^{4} / 10\right)$ in units where $\hbar=1 . E_{n}^{\#}$ are the highly accurate numerical values of Ref. 10. $E_{n}$ are the values obtained using the zero order variational Sturmian approximation. $E_{n}^{\mathrm{CSA}}$ are the values obtained by the zero order conventional Sturmian approximation in Ref. 5. $E_{n}^{(0)}$ and $E_{n}^{(1)}$ are the energy eigenvalues obtained using the zero and first order perturbation theory, respectively.

\begin{tabular}{cccccccccc}
\hline \hline$n$ & $E_{n}^{\#}$ & $E_{n}$ & $\frac{\left|E_{n}-E_{n}^{\#}\right|}{E_{n}^{\#}}$ & $E_{n}^{\mathrm{CSA}}$ & $\frac{\left|E_{n}^{\mathrm{CSA}}-E_{n}^{\#}\right|}{E_{n}^{\#}}$ & $E_{n}^{(0)}$ & $\frac{\left|E_{n}^{(0)}-E_{n}^{\#}\right|}{E_{n}^{\#}}$ & $E_{n}^{(1)}$ & $\frac{\left|E_{n}^{(1)}-E_{n}^{\#}\right|}{E_{n}^{\#}}$ \\
\hline 0 & 1.065286 & 1.06692 & $1.5 \times 10^{-3}$ & 1.07500 & $9.1 \times 10^{-3}$ & 1.000 & 0.061 & 1.075 & $9.1 \times 10^{-3}$ \\
1 & 3.306872 & 3.31182 & $1.5 \times 10^{-3}$ & 3.37500 & 0.021 & 3.000 & 0.032 & 3.450 & 0.043 \\
2 & 5.747959 & 5.75052 & $4.5 \times 10^{-4}$ & 5.97500 & 0.040 & 5.000 & 0.13 & 5.975 & 0.039 \\
3 & 8.352678 & 8.34985 & $3.4 \times 10^{-4}$ & 8.87500 & 0.063 & 7.000 & 0.16 & 8.875 & 0.063 \\
4 & 11.09860 & 11.0881 & $9.5 \times 10^{-4}$ & 12.0750 & 0.088 & 9.000 & 0.19 & 12.08 & 0.088 \\
5 & 13.96993 & 13.9499 & $1.4 \times 10^{-3}$ & & & 11.00 & 0.21 & 15.58 & 0.11 \\
6 & 16.95479 & 16.9235 & $1.8 \times 10^{-3}$ & & & 13.00 & 0.23 & 19.38 & 0.14 \\
7 & 20.04386 & 19.9998 & $2.2 \times 10^{-3}$ & & & 15.00 & 0.25 & 23.48 & 0.17 \\
8 & 23.22955 & 23.1715 & $2.5 \times 10^{-3}$ & & & 17.00 & 0.27 & 27.88 & 0.20 \\
9 & 26.50555 & 26.4322 & $2.8 \times 10^{-3}$ & & & 19.00 & 0.28 & 32.58 & 0.23 \\
\hline \hline
\end{tabular}

where

$$
r_{n}:=\frac{4 p_{n}^{3}}{27 q_{n}^{2}}=\left(\frac{8 n+4}{11 n^{2}+9 n+4}\right)^{2} r_{0}, \quad r_{0}:=\frac{m k^{3}}{108 \hbar^{2} \epsilon^{2}} .
$$

The right-hand side of Eq. (77) is manifestly real and positive for $r_{n} \leqslant 1$. It is not difficult to check that it is also real and positive for $r_{n}>1$. In fact, we can express $\mathcal{E}$ in the form

$$
\mathcal{E}=2 \sqrt{\frac{p_{n}}{3}} \cos \left(\frac{\phi_{n}}{3}\right)=\sqrt{\frac{k}{3 m}}(2 n+1) \cos \left(\frac{\phi_{n}}{3}\right),
$$

where

$$
\phi_{n}:=\tan ^{-1}\left(\sqrt{r_{n}-1}\right) .
$$

Note that for $r_{n}<1, \phi_{n}$ is imaginary, but $\cos \left(\phi_{n} / 3\right)$ is still real and positive.

Having fixed the parameter $\mathcal{E}$, we can determine the energy eigenvalues $E_{n}$ using Eqs. (43) and (72). We first use Eqs. (37) and (79) to compute

$$
\alpha_{n}=\left(\frac{2}{\hbar}\right) \sqrt{\frac{m k}{3}} \cos \left(\frac{\phi_{n}}{3}\right) .
$$

Then substituting this equation in Eq. (72) and using Eq. (43), we find

$$
E_{n}=\left(\frac{\hbar}{24}\right) \sqrt{\frac{3 k}{m}}(2 n+1)\left[7+3 \tan ^{2}\left(\frac{\phi_{n}}{3}\right)\right] \cos \left(\frac{\phi_{n}}{3}\right)+\left(\frac{9 \hbar^{2} \epsilon}{16 m k}\right)\left(2 n^{2}+2 n+1\right)\left[1+\tan ^{2}\left(\frac{\phi_{n}}{3}\right)\right] .
$$

In particular, the ground state energy is given by

$$
E_{0}=\left(\frac{\hbar}{24}\right) \sqrt{\frac{3 k}{m}}\left[7+3 \tan ^{2}\left(\frac{\phi_{0}}{3}\right)\right] \cos \left(\frac{\phi_{0}}{3}\right)+\left(\frac{9 \hbar^{2} \epsilon}{16 m k}\right)\left[1+\tan ^{2}\left(\frac{\phi_{0}}{3}\right)\right] .
$$

In Table I, we list the numerical values obtained using Eq. (82) for the first 10 energy levels of a quartic anharmonic oscillator with $m=1 / 2, k=2, \epsilon=1 / 10$ in units where $\hbar=1$. This table also 
TABLE II. Energy levels of the Hamiltonian $H=p^{2}+x^{2}+x^{4} / 10$ obtained using the first order variational Sturmian approximation. $\delta E_{n}$ stands for $\left|E_{n}-E_{n}^{\#}\right| / E_{n}^{\#}$.

\begin{tabular}{lcccccccccc}
\hline \hline $\mathcal{S}_{2}$ & $E_{0}$ & $\delta E_{0}$ & $E_{1}$ & $\delta E_{1}$ & $E_{2}$ & $\delta E_{2}$ & $E_{3}$ & $\delta E_{3}$ & $E_{4}$ & $\delta E_{4}$ \\
\hline$\{0,2\}$ & 1.06614 & $8.0 \times 10^{-4}$ & & & 5.76117 & $2.3 \times 10^{-3}$ & & & & \\
$\{1,3\}$ & & & 3.30922 & $7.1 \times 10^{-4}$ & & & 8.37284 & $2.4 \times 10^{-3}$ & & \\
$\{2,4\}$ & & & & & 5.74558 & $4.1 \times 10^{-4}$ & & & 9.66370 & 0.13 \\
$\{0,4\}$ & 1.06620 & $8.6 \times 10^{-4}$ & & & & & & & 9.64502 & 0.13 \\
\hline
\end{tabular}

includes the accurate numerical values of Ref. 10, the values obtained using the conventional Sturmian approximation and the zero and first order perturbation theory. [The zero and first order perturbation theory yield

$$
E_{n}^{(0)}=\hbar(k / m)^{1 / 2}(n+1 / 2), \quad E_{n}^{(1)}=E_{n}^{(0)}+3 \hbar^{2} \epsilon^{2}\left(2 n^{2}+2 n+1\right) /(4 m k),
$$

respectively.] The relative difference between the results of the variational Sturmian approximation of order zero with the highly accurate numerical results $\left(E_{n}^{\#}\right)$ of Ref. 10, i.e., the quantity $\left|E_{n}-E_{n}^{\#}\right| / E_{n}^{\#}$, varies between $3.38 \times 10^{-4}$ and $2.77 \times 10^{-3}$. For the ground state, this number is $1.53 \times 10^{-3}$. Even for the lowest lying energy levels where perturbation theory yields reliable results, the zero order variational Sturmian approximation produces more accurate values than both the zero and first order perturbation theory. As seen from Table I, the variational Sturmian approximation is better than the conventional Sturmian approximation.

In the remainder of this section we present the results obtained using the first and second order variational Sturmian approximation. The numerical results are respectively presented in Tables II and III.

As we explained in Sec. IV, in the variational Sturmian approximation of order one one chooses an indexing set $\mathcal{S}_{2}$ consisting of two Sturmians to be included in the expansion of the eigenvector $|E\rangle$. One then solves the corresponding secular equation (19), expresses the solutions $E_{ \pm}$in terms of the parameter $\mathcal{E}$, and finds the minima of $E_{ \pm}(\mathcal{E})$. In general, $E_{ \pm}$are complicated functions of $\mathcal{E}$. However, it turns out that for all the cases that we considered $E_{ \pm}$has a unique minimum corresponding to a positive value of $\mathcal{E}$.

In order to choose the indexing set $\mathcal{S}_{2}$, we first note that the Sturmian functions $\left\langle x \mid \phi_{n}\right\rangle$ with even (respectively, odd) $n$ are even (respectively odd) functions of $x$. We expect the energy eigenfunctions of the anharmonic oscillator (70) to have the same parity structure as the Sturmian functions. This, in particular, suggests that in the calculation of $E_{0}$ we should take $\mathcal{S}_{2}=\{0,2\}$.

For a quartic anharmonic oscillator with $m=1 / 2, k=2, \epsilon=1 / 10$, the first order variational Sturmian approximation corresponding to $\mathcal{S}_{2}=\{0,2\}$ yields $E_{0}=E_{-}=1.06614$ and $E_{2}=E_{+}$ $=5.76117$. The value obtained for $E_{0}$ differs from the accurate numerical value by one part in $10^{4}$. It is one order of magnitude better than the value obtained using the zero order variational Sturmian approximation. The value for $E_{2}$ is however less accurate. One may argue that the choice made for $\mathcal{S}_{2}$ is appropriate only for the ground state. In order to compute $E_{2}$ using the first order

TABLE III. Energy levels of the Hamiltonian $H=p^{2}+x^{2}+x^{4} / 10$ obtained using the second order variational Sturmian approximation with the choice $\{0,2,4\}$ for the indexing set $\mathcal{S}_{3} . E_{n}^{\#}$ are the highly accurate numerical values of Ref. 10.

\begin{tabular}{cccl}
\hline \hline$n$ & $E_{n}^{\#}$ & $E_{n}$ & $\frac{\left|E_{n}-E_{n}^{\#}\right|}{E_{n}^{\#}}$ \\
\hline 0 & 1.065286 & 1.06613 & $7.9 \times 10^{-4}$ \\
2 & 5.75052 & 5.75275 & $8.3 \times 10^{-4}$ \\
4 & 11.09860 & 9.68483 & 0.127 \\
\hline \hline
\end{tabular}


variational Sturmian approximation, one may alternatively choose $\mathcal{S}_{2}=\{2,4\}$. This choice yields $E_{2}=E_{-}=5.74558$ and $E_{4}=E_{+}=9.6637$. Again this value for $E_{2}$ is an order of magnitude better than the value obtained using the zero order variational Sturmian approximation, whereas the value for $E_{4}$ is less accurate. One can also try $\mathcal{S}_{2}=\{0,4\}$. As expected, this choice yields a less accurate value than the choices $\mathcal{S}_{2}=\{0,2\}$ and $\mathcal{S}_{2}=\{2,4\}$ for both $E_{0}$ and $E_{4}$.

For the calculation of the first excited state we choose $\mathcal{S}_{2}=\{1,3\}$. Then we find $E_{1}=E_{-}$ $=3.30922$ and $E_{3}=E_{+}=8.37284$. Once again the first order variational Sturmian approximation of order one with the choice $\mathcal{S}_{2}=\{1,3\}$ yields a more accurate result for $E_{1}$ and a less accurate result for $E_{3}$.

In general, in the calculation of the energy levels $E_{n}$ with $n \geqslant 2$, there are two alternative choices for the indexing set $\mathcal{S}_{2}$. In view of the parity properties of the eigenvectors, these are $\{n, n+2\}$ and $\{n-2, n\}$. The fact that there is no physical reason to distinguish between these two choices suggests that for these levels one should consider the second order variational Sturmian approximation with the choice $\mathcal{S}_{3}=\{n-2, n, n+2\}$.

Table III includes the results of the second order variational Sturmian approximation corresponding to the indexing set $\mathcal{S}_{3}=\{0,2,4\}$. This approximation yields more accurate values for $E_{0}$ than the zero and first order variational Sturmian approximations. However, contrary to our expectation the value obtained for $E_{2}$ is less accurate than the one given by the zero order approximation and the first order approximation with $\mathcal{S}_{2}=\{2,4\}$.

\section{B. The quartic potential}

Consider the quartic potential

$$
V(x)=\epsilon x^{4} .
$$

We can easily obtain the energy levels of this potential using the zero order variational Sturmian approximation by simply setting $k=0$ in our formulas for the quartic anharmonic oscillator. Substituting $k=0$ in (76), we can write Eq. (75) in the form

$$
\mathcal{E}=q_{n}^{1 / 3}=\hbar\left(\frac{\hbar \epsilon}{2 m^{2}}\right)^{1 / 3}\left[\left(n+\frac{1}{2}\right)^{2}\left(11 n^{2}+9 n+4\right)\right]^{1 / 3} .
$$

In view of Eqs. (37), (43), (72), (86), and $k=0$, we have

$$
\begin{gathered}
\alpha_{n}=\hbar^{-1}(m \hbar \epsilon)^{1 / 3}\left(\frac{11 n^{2}+9 n+4}{2 n+1}\right)^{1 / 3}, \\
E_{n}=\left(\frac{8 n+4}{11 n^{2}+9 n+4}\right)^{2 / 3}\left(\frac{17}{7} n^{2}+\frac{15}{7} n+1\right) E_{0}, \\
E_{0}:=\frac{7 \hbar}{8}\left(\frac{\hbar \epsilon}{2 m^{2}}\right)^{1 / 3} .
\end{gathered}
$$

In Table IV, we present the values obtained using Eq. (88) for the energy levels of a quartic potential with $m=1 / 2$ and $\epsilon=1$ in units where $\hbar=1$. This table also includes accurate numerical results $E_{n}^{\#}$ and the results of the zero and first order WKB approximation given in Refs. 11 and 12. The relative difference $\left|E_{n}-E_{n}^{\#}\right| / E_{n}^{\#}$ is about 0.04 for the ground state and ranges between 6.4 $\times 10^{-4}$ and $8.7 \times 10^{-3}$ for the energy levels $E_{2}, E_{4}, E_{6}, E_{8}, E_{10}$, and $E_{16}$.

Table $\mathrm{V}$ includes the results of the first and second order variational Sturmian approximation for $E_{0}, E_{2}$, and $E_{4}$. 
TABLE IV. Energy levels of the Hamiltonian $H=p^{2}+x^{4}$ in units where $\hbar=1 . E_{n}^{\#}$ are the highly accurate numerical values of Refs. 11 and 12. $E_{n}$ are the values obtained using the zero order variational Sturmian approximation. $E_{n}^{\mathrm{WKB}(0)}$ and $E_{n}^{\mathrm{WKB}(1)}$ are the values obtained using the zero and first order WKB approximation (Ref. 11), respectively.

\begin{tabular}{cccccccc}
\hline \hline$n$ & $E_{n}^{\#}$ & $E_{n}$ & $\frac{\left|E_{n}-E_{n}^{\#}\right|}{E_{n}^{\#}}$ & $E_{n}^{(0)}$ & $\frac{\left|E_{n}^{\mathrm{WKB}(0)}-E_{n}^{\#}\right|}{E_{n}^{\#}}$ & $E_{n}^{(1)}$ & $\frac{\left|E_{n}^{\mathrm{WKB}(1)}-E_{n}^{\#}\right|}{E_{n}^{\#}}$ \\
\hline 0 & 1.060362 & 1.10243 & 0.040 & 0.87 & 0.17 & 0.98 & 0.076 \\
1 & & 3.86929 & & & & & \\
2 & 7.455697 & 7.46048 & $6.4 \times 10^{-4}$ & 7.4140 & $5.6 \times 10^{-3}$ & 7.4558 & $1.4 \times 10^{-5}$ \\
3 & & 11.6007 & & & & & \\
4 & 16.261826 & 16.1691 & $5.7 \times 10^{-3}$ & 16.233615 & $1.7 \times 10^{-3}$ & 16.261937 & $6.8 \times 10^{-6}$ \\
6 & 26.528471 & 26.3349 & $7.3 \times 10^{-3}$ & 26.506336 & $8.3 \times 10^{-4}$ & 26.528513 & $1.9 \times 10^{-5}$ \\
8 & 37.923001 & 37.6218 & $7.9 \times 10^{-3}$ & 37.904472 & $4.9 \times 10^{-4}$ & 37.923021 & $5.3 \times 10^{-7}$ \\
10 & 50.256255 & 49.8404 & $8.3 \times 10^{-3}$ & 50.240152 & $3.1 \times 10^{-4}$ & 50.256266 & $2.2 \times 10^{-7}$ \\
16 & 91.79806 & 91.0012 & $8.7 \times 10^{-3}$ & & & & \\
\hline \hline
\end{tabular}

\section{The Gaussian potential}

Consider the Gaussian potential

$$
V(x)=-\lambda e^{-\epsilon x^{2} / 2}
$$

In order to apply the results of Sec. IV to this potential, we write $V(x)=\widetilde{V}(x)-\lambda$ where

$$
\widetilde{V}(x)=\lambda\left(1-e^{-\epsilon x^{2} / 2}\right) .
$$

Then as $\epsilon$ tends to zero, $\widetilde{V}(x)$ approaches to the harmonic oscillator potential (27) with $k=\lambda \epsilon$.

Clearly, the energy eigenvalues associated with $V$ and $\widetilde{V}$ are related by

$$
E_{n}=\widetilde{E}_{n}-\lambda
$$

In the following we use the zero order variational Sturmian approximation to obtain the ground state energy of the potential $\widetilde{V}$. The excited energy levels can be obtained similarly.

We first note that for the ground state $n=0$, and Eq. (50) for the potential $\widetilde{V}$ takes the form

$$
\mathcal{E}=\sqrt{2}{ }_{0}\langle 0|\tilde{V}| 2\rangle_{0}=\sqrt{2}{ }_{0}\langle 0|V| 2\rangle_{0}=-\sqrt{2} \lambda_{0}\left\langle 0\left|e^{-\epsilon x^{2} / 2}\right| 2\right\rangle_{0}=-\sqrt{2} \lambda \int_{-\infty}^{\infty}{ }_{0}\langle 0 \mid x\rangle e^{-\epsilon x^{2} / 2}\langle x \mid 2\rangle_{0} d x .
$$

We can evaluate the right-hand side of (93) using the well-known expression for the eigenfunctions of the harmonic oscillator, namely (32) and

$$
\langle x \mid 2\rangle_{0}=\left(\frac{\alpha_{0}}{4 \pi}\right)^{1 / 4}\left(2 \alpha_{0} x^{2}-1\right) e^{-\alpha_{0} x^{2} / 2} .
$$

TABLE V. Energy levels of the Hamiltonian $H=p^{2}+x^{4}$ obtained using the first and second order variational Sturmian approximation. $N$ is the order of the approximation. $E_{n}^{\#}$ are the accurate numerical results reported in Ref. 11.

\begin{tabular}{rrrrclll}
\hline \hline$N$ & $\mathcal{S}_{N}$ & $E_{0}$ & $\frac{\left|E_{0}-E_{0}^{\#}\right|}{E_{0}^{\#}}$ & $E_{2}$ & $\frac{\left|E_{2}-E_{2}^{\#}\right|}{E_{2}^{\#}}$ & \multicolumn{1}{c}{$E_{4}$} & $\frac{\left|E_{4}-E_{4}^{\#}\right|}{E_{4}^{\#}}$ \\
\hline 2 & $\{0,2\}$ & 1.08110 & 0.0196 & 7.60884 & 0.0205 & & \\
2 & $\{2,4\}$ & & & 7.42669 & $3.89 \times 10^{-3}$ & 16.4461 & 0.0113 \\
2 & $\{0,4\}$ & 1.08166 & 0.0200 & & & 16.4114 & $9.12 \times 10^{-3}$ \\
3 & $\{0,2,4\}$ & 1.08010 & 0.0195 & 7.56528 & 0.0147 & 16.5670 & 0.0188 \\
\hline \hline
\end{tabular}


Substituting Eqs. (32) and (94) in Eq. (93) and performing the necessary calculations, we find

$$
\mathcal{E}(\mathcal{E}+p)^{3}=\lambda^{2} p^{2}
$$

where

$$
p:=\frac{\hbar^{2} \epsilon}{4 m} .
$$

Introducing

$$
\eta:=1+\frac{\mathcal{E}}{p}
$$

we can write Eq. (95) in the form

$$
f(\eta):=\eta^{4}-\eta^{3}-r=0
$$

where

$$
r:=\frac{\lambda^{2}}{p^{2}}=\frac{16 \lambda^{2} m^{2}}{\hbar^{4} \epsilon^{2}} .
$$

It is not difficult to show that for all $r>0, f(\eta)$ has a single minimum at $\eta=3 / 4$. The minimum is $f(3 / 4)=-(27 / 256+r)<0$. Furthermore, $f(0)=f(1)=-r<0$ and $\lim _{\eta \rightarrow \infty} f(\eta)=\infty$. Therefore, $f(\eta)$ has a single positive root that is greater than 1 . This root is given by

$$
\eta_{\star}=\frac{1}{4}\left(1+2 \xi+\sqrt{3-4 \xi^{2}+\xi^{-1}}\right),
$$

where

$$
\begin{aligned}
& \xi:=\frac{1}{2} \sqrt{1-a+b}, \quad a:=\frac{3}{2} \zeta\left(1+\sqrt{1+\zeta^{3}}\right)^{1 / 3}, \\
& b:=\frac{3}{2} \zeta\left(-1+\sqrt{1+\zeta^{3}}\right)^{1 / 3}, \quad \zeta:=\frac{4}{3}(4 r)^{1 / 3} .
\end{aligned}
$$

In view of Eq. (97) and the fact that $\eta_{0}>1$, Eq. (95) has a single positive solution, namely

$$
\mathcal{E}=p\left(\eta_{\star}-1\right)
$$

Having obtained the parameter $\mathcal{E}$, we next compute

$$
W={ }_{0}\langle 0|\tilde{V}| 0\rangle_{0}=\lambda\left(1-\sqrt{1-\eta_{\star}^{-1}}\right) .
$$

Here we have made use of Eqs. (32), (37), (91), (96), and (101). Substituting this equation and Eq. (101) in Eq. (43) and using Eqs. (92) and (99), we find the ground state energy of the Gaussian potential $(90)$ to be

$$
E_{0}=\widetilde{E}_{0}-\lambda=-\lambda\left[\sqrt{1-\eta_{\star}^{-1}}+\frac{1-\eta_{\star}}{2 \sqrt{r}}\right]
$$

In order to reveal the asymptotic behavior of $E_{0}$, we investigate the power series expansion of the right-hand side of Eq. (103).

For $r \gg 1$, i.e., $(\epsilon / \lambda) \rightarrow 0$, 


$$
E_{0}=-\lambda\left[1-r^{-1 / 4}+\frac{3}{8} r^{-1 / 2}-\frac{1}{32} r^{-3 / 4}-\frac{1}{128} r^{-1}+\mathcal{O}\left(r^{-5 / 4}\right)\right]
$$

For $r \ll 1$, i.e., $(\epsilon / \lambda) \rightarrow \infty$,

$$
E_{0}=-\left(\frac{\lambda \sqrt{r}}{2}\right)\left[1-r+3 r^{2}-13 r^{3}+68 r^{4}+\mathcal{O}\left(r^{5}\right)\right] .
$$

Therefore, for fixed $\lambda$,

$$
\begin{gathered}
\lim _{\epsilon \rightarrow 0^{+}} E_{0}=-\lambda, \\
\lim _{\epsilon \rightarrow \infty} E_{0}=\lim _{\epsilon \rightarrow \infty}\left[-\frac{4 m^{2} \lambda^{2}}{\hbar^{2} \epsilon}\right]=0^{-},
\end{gathered}
$$

and for fixed $\epsilon$,

$$
\begin{gathered}
\lim _{\lambda \rightarrow 0^{+}} E_{0}=\lim _{\lambda \rightarrow 0}\left[-\frac{4 m^{2} \lambda^{2}}{\hbar^{2} \epsilon}\right]=0^{-}, \\
\lim _{\lambda \rightarrow \infty} E_{0}=-\infty .
\end{gathered}
$$

Clearly, the asymptotic behavior of $E_{0}$, as given by Eqs. (106)-(109), agrees with the qualitative analysis of the eigenvalue problem for the Gaussian potential.

It is not difficult to see that in the limit $\epsilon \rightarrow 0$ perturbation theory provides reliable results. Writing the Gaussian potential (90) in the form

$$
V=V_{0}+\delta V
$$

with $V_{0}$ given by Eq. (23) and performing the standard calculations, ${ }^{1}$ we find that the zero and first order perturbation theory yield, respectively,

$$
\begin{gathered}
E_{0}^{(0)}=-\lambda\left(1-r^{-1 / 4}\right), \\
E_{0}^{(1)}=-\lambda\left(1+r^{-1 / 4}\right)^{-1 / 2}=-\lambda\left[1-\frac{1}{2} r^{-1 / 4}+\frac{3}{8} r^{-1 / 2}-\frac{5}{16} r^{-3 / 4}+\mathcal{O}\left(r^{-1}\right)\right] .
\end{gathered}
$$

Here $E_{0}^{(l)}$ is the ground state energy for the Gaussian potential (90) obtained using the $l$ th order perturbation theory.

Comparing Eq. (104) with Eqs. (111) and (112), one finds that in the pertubative region where $r \gg 1$, the variational Sturmian approximation of order zero agrees with the results of the perturbation theory. In fact, since by construction $E_{0}$ is the expectation value of the energy of the Sturmian $\left|\phi_{0}\right\rangle$, the fact that $E_{0}<E_{0}^{(0)}$ shows that even in the pertubative region the variational Sturmian approximation of order zero is a better approximation than the zero order perturbation theory. By the same reasoning, because $E_{0}>E_{0}^{(1)}$, the first order perturbation theory yields a better result. Note however that the wave function obtained in the first order perturbation theory is an infinite sum whereas the wave function in the zero order Sturmian approximation is given explicitly.

Another interesting limit is the delta function limit of the potential $V$ where $\lambda=a \sqrt{\epsilon /(2 \pi)}$, $\epsilon \rightarrow \infty$, and $V(x) \rightarrow-a \delta(x)$. Here $a$ is a fixed coupling constant. In this limit $r \rightarrow 0$ and the ground state energy is given by Eq. (105) according to

$$
E_{0}=-\frac{m a^{2}}{\pi \hbar^{2}}
$$


This result has the same order of magnitude as the exact result:

$$
E_{0}=-\frac{m a^{2}}{2 \hbar^{2}}
$$

\section{DISCUSSION AND CONCLUSION}

We have outlined a variationally improved Sturmian approximation and applied our results to the harmonic oscillator Sturmians. For these Sturmians we could solve the associated variational problem in the zero order Sturmian approximation exactly. We have used our variational Sturmian approximation in the calculation of the energy levels of various potentials. We have shown that using a few harmonic oscillator Sturmians, one obtains quite reliable results. In general, the variational Sturmian approximation is a better approximation than the conventional Sturmian approximation.

Because the harmonic oscillator potential is a confining potential, we expect that the method is more suitable for the confining potentials such as the quartic anharmonic oscillator and the quartic potential. We can base this argument on a more quantitative reasoning by addressing the problem of classifying the potentials for which the Sturmian approximation is exact. It is not difficult to show that these potentials satisfy

$$
V(\vec{x})=E-\mathcal{E}+\left(\frac{\sum_{\nu=0}^{N} \sum_{\alpha} C_{\nu}^{\alpha} \beta_{\nu} \phi_{\nu, \alpha}(\vec{x})}{\sum_{\nu=0}^{N} C_{\nu}^{\alpha} \phi_{\nu, \alpha}(\vec{x})}\right) V_{0}(\vec{x}),
$$

where $E, \mathcal{E}$, and $C_{\nu}^{\alpha}$ are constants and $\phi_{\nu, \alpha}(\vec{x}):=\left\langle\vec{x} \mid \phi_{\nu}, \alpha\right\rangle$. Equation (115) follows from Eqs. (1), (2), (3), and (11).

For example, the potentials for which the first order harmonic oscillator Sturmian approximation with $\mathcal{S}_{2}=\{0,2\}$ yields an exact eigenfunction are of the form

$$
V(x)=E-\frac{\hbar^{2} \alpha_{0}}{2 m}+\left(\frac{\hbar^{2} \alpha_{0}^{2}}{2 m}\right)\left[\frac{e^{-2 \alpha_{0} x^{2} / 5}+\left(\frac{\zeta}{5}\right)\left(2 \alpha_{0} x^{2}-5\right)}{e^{-2 \alpha_{0} x^{2} / 5}+5 \zeta\left(2 \alpha_{0} x^{2}-5\right)}\right] x^{2},
$$

where $\alpha_{0}$ is a real parameter with the dimension of (length) ${ }^{-2}$ and $\zeta$ is a dimensionless real parameter. As seen from Eq. (116), these potentials tend to the harmonic oscillator potential for $|x| \rightarrow \infty$. In particular, as $|x| \rightarrow \infty, V \rightarrow \infty$. This asymptotic behavior is also valid for higher order harmonic oscillator Sturmian approximations. This observation shows that the harmonic oscillator Sturmian approximation is more reliable for confining potentials.

We conclude this paper with a couple of remarks.

(1) The variational principle used in the variational Sturmian approximation leads to an algebraic (nondifferential) equation for the parameter $\mathcal{E}$. The acceptable solutions for this equation are those which are real and positive. The fact that for all the cases we consider there is a unique real positive solution corresponding to each eigenvalue $E_{n}$ is quite remarkable. This observation may be viewed as a consistency check for the Sturmian approximation.

(2) In our selection of the Sturmians in the first and higher order Sturmian approximation, we used the information about the parity properties of the Sturmians and the energy eigenfunctions. For example we ruled out the first order variational Sturmian approximation with $\mathcal{S}_{2}=\{0,1\}$. If we perform the necessary calculations, we find that for this choice the functions $t$ and $w$ vanish identically and the matrices $T$ and $S$ are diagonal. Therefore, the secular equation (19) yields the same results as the zero order Sturmian approximation. This can also be seen from the results of Ref. 5. 
${ }^{1}$ D. Bohm, Quantum Theory (Prentice-Hall, New York, 1951); J. J. Sakurai, Modern Quantum Mechanics (AddisonWesley, Reading, MA, 1994).

${ }^{2}$ L. D. Landau and E. M. Lifshitz, Quantum Mechanics (Butterworth Heinemann, Oxford, 1977).

${ }^{3}$ M. Rotenberg, Ann. Phys. (N.Y.) 19, 262 (1962).

${ }^{4}$ J. Avery and D. R. Herschbach, Int. J. Quantum Chem. 41, 673 (1992); J. Avery, J. Math. Chem. 21, 285 (1997); J. Avery and F. Antonsen, ibid. 24, 175 (1998); J. Avery, Adv. Quantum Chem. 31, 201 (1999); J. Mol. Struct. 458, 1 (1999); R. Szmytkowski, J. Phys. A 33, 427 (2000).

${ }^{5}$ F. Antonsen, Phys. Rev. A 60, 812 (1999).

${ }^{6}$ R. Szmytkowski and B. Zywicka-Mozeiko, Phys. Rev. A 62, 2104 (2000).

${ }^{7}$ A. Messiyah, Quantum Mechanics (North-Holland, Amsterdam, 1964).

${ }^{8}$ E. J. Weniger, J. Math. Phys. 26, 276 (1985).

${ }^{9}$ H. M. Edwards, Galois Theory (Springer-Verlag, New York, 1984).

${ }^{10}$ B. Bacus, Y. Meurice, and A. Soemadi, J. Phys. A 28, L381 (1995).

${ }^{11}$ C. M. Bender, K. Olaussen, and P. S. Wang, Phys. Rev. D 16, 1740 (1977).

${ }^{12}$ A. Voros, J. Phys. A 27, 4653 (1994). 